\title{
Continuum theory of critical phenomena in polymer solutions: Formalism and mean field approximation
}

\author{
Raymond E. Goldstein \\ Laboratory of Atomic and Solid State Physics and Materials Science Center, Clark Hall, \\ Cornell University, Ithaca, New York 14853-2501 and Service de Physique Théorique, \\ Centre d'Etudes Nucléaires de Saclay, F-91191 Gif-sur-Yvette Cedex, France and The Enrico Fermi \\ Institute, The University of Chicago," ${ }^{\text {) }}$ Chicago, Illinois 60637 \\ Binny J. Cherayil \\ Department of Chemistry and Baker Laboratory, Cornell University, Ithaca, New York 14853-1301
}

(Received 17 October 1988; accepted 14 February 1989)

\begin{abstract}
A theoretical description of the critical point of a polymer solution is formulated directly from the Edwards continuum model of polymers with two- and three-body excluded-volume interactions. A Hubbard-Stratonovich transformation analogous to that used in recent work on the liquid-vapor critical point of simple fluids is used to recast the grand partition function of the polymer solution as a functional integral over continuous fields. The resulting LandauGinzburg-Wilson (LGW) Hamiltonian is of the form of a generalized nonsymmetric $n=1$ component vector model, with operators directly related to certain connected correlation functions of a reference system. The latter is taken to be an ensemble of Gaussian chains with three-body excluded-volume repulsions, and the operators are computed in three dimensions by means of a perturbation theory that is rapidly convergent for long chains. A mean field theory of the functional integral yields a description of the critical point in which the powerlaw variations of the critical polymer volume fraction $\phi_{c}$, critical temperature $T_{c}$, and critical amplitudes on polymerization index $N$ are essentially identical to those found in the FloryHuggins theory. In particular, we find $\phi_{c} \sim N^{-1 / 2}, T_{\theta}-T_{c} \sim N^{-1 / 2}$ with $\left(T_{\theta}\right.$ the theta temperature), and that the composition difference between coexisting phases varies with reduced temperature $t$ as $N^{-1 / 4} t^{1 / 2}$. The mean field theory of the interfacial tension $\sigma$ between coexisting phases near the critical point, developed by considering the LGW Hamiltonian for a weakly inhomogeneous solution, yields $\sigma \sim N^{-1 / 4} t^{3 / 2}$, with the correlation length diverging as $\xi \sim N^{1 / 4} t^{-1 / 2}$ within the same approximation, consistent with the mean field limit of de Gennes' scaling form. Generalizations to polydisperse systems are discussed.
\end{abstract}

\section{INTRODUCTION AND SUMMARY OF RESULTS}

The critical behavior of polymer solutions differs from that of simple liquids in exhibiting the phenomenon of universality not only in the exponents characterizing singularities in thermodynamic properties but also in their amplitudes. Thus, for any property $P$ that is a function, say, of temperature $T$, and that near the critical point varies with the reduced temperature $t=\left|T-T_{c}\right| / T_{c}$ as

$$
P(T) \simeq P_{0}(N) t^{\psi_{P}},
$$

with $\psi_{p}$ universal, it is known from both theory and experiment that the amplitude $P_{0}$ exhibits a universal power-law dependence on the polymer molecular weight $N$,

$$
P_{0}(N) \simeq P_{0}^{\prime} N^{-\zeta_{P}}
$$

$P_{0}^{\prime}$ being nonuniversal.

To describe the nature of this additional polymer universality, we present in Fig. 1 a schematic illustration of the temperature-composition phase diagram of a binary mixture of long-chain polymers in a solvent and focus on the molecular weight dependence of several important properties. First, consider the order parameter of the phase transition, conve-

\footnotetext{
a) Present address.
}

niently taken to be the difference in the compositions $\phi^{\prime}$ and $\phi^{\prime \prime}$ between coexisting phases. This quantity is known to vary with the same singular dependence on reduced tem-

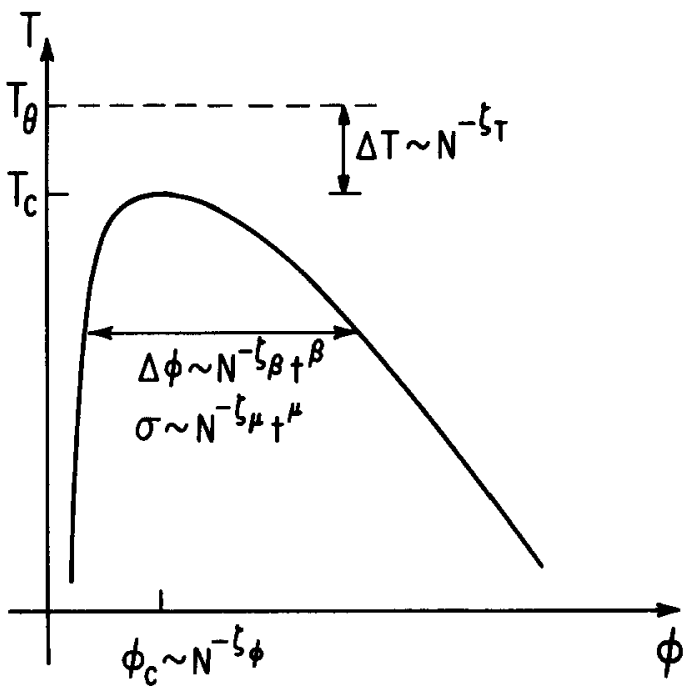

FIG. 1. The neighborhood of the critical point in the temperature-volume fraction phase diagram of a polymer solution. Power-law variations with reduced temperature $t=\left(T_{c}-T\right) / T_{c}$ and molecular weight $N$ are indicated. 
perature $t$ that is found in simple binary mixtures and pure fluids, but, additionally, it shows a power-law dependence on $N,^{1}$

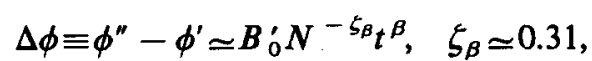

with $\beta \simeq 0.33$. Similarly, the surface tension between coexisting phases varies as ${ }^{2}$

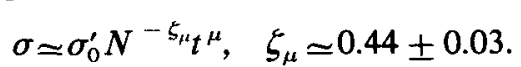

Like $\beta$, the thermal exponent $\mu(\simeq 1.26)$ also governs the critical properties of simple fluids and mixtures. Other quantities, not related to critical singularities themselves, also exhibit universal power laws in the molecular weight. An example is the volume fraction $\phi_{c}$ of polymer at the critical point itself, which is known from recent experiments ${ }^{2.3}$ to vary as

$$
\phi_{c} \simeq \phi_{0} N^{-\xi_{\phi}}, \quad \zeta_{\phi} \simeq 0.40 \pm 0.02
$$

The existence of universal critical amplitudes may be related to the existence of a scaling form of the singular part of the free energy density $g_{s}$ in the neighborhood of the critical point. As is the case in simple fluids and mixtures, one expects $g_{s}$ to have the form

$$
g_{s}(T, \mu) \simeq D(N) \tau^{2-\alpha} Y\left[E(N) \frac{\zeta}{\tau^{\beta \delta}}\right],
$$

with $D(N)$ and $E(N)$ nonuniversal metric coefficients, $\tau$ and $\zeta$ the "thermal" and "magnetic" scaling fields, $\alpha, \beta$, and $\delta$ the universal exponents, and $Y$ the universal scaling function of a system in the universality class of an Ising model in three dimensions. The exponents $\zeta$ of the individual critical properties will then be determined by the corresponding universal power-law variations of the metric coefficients $D$ and $\boldsymbol{E}$.

The values for the various indices $\xi$ deviate significantly from those predicted by mean field theories of polymer solutions, such as the Flory-Huggins ${ }^{4}$ theory, which gives $\zeta_{\beta}$ $=1 / 4$ and $\zeta_{\phi}=1 / 2$. The surface tension exponent $\zeta_{\mu}$ shows a very marked deviation from the mean field predictions of Vrij and Roebersen ${ }^{5}$ and of Nose, ${ }^{6}$ namely $\zeta_{\mu}=1 / 4$. One should note that the existence of universal amplitude ratios governing Ising-like critical points enforces relationships between the various exponents $\zeta{ }^{7}$ and these are in general supported by experiment, but it remains an important open problem in the statistical mechanics of polymeric fluids to develop a microscopic theory of the power-law variations of critical properties with molecular weight.

To understand these discrepancies between mean field theory and experiment it is desirable that there be a theoretical formalism through which contact may be made with the powerful field-theoretic methods available to study critical phenomena. For the latter purpose, it is most convenient to recast the singular contribution to the partition function as a functional integral over continuous fields,

$$
\Xi_{s} \propto \int \mathscr{D}[\varphi] \exp \left(-\mathscr{H}_{\mathrm{LGW}}[\varphi]\right)
$$

Given that the polymer critical solution point is that of an Ising-like system, one expects, in the long-wavelength limit, the Landau-Ginzburg-Wilson (LGW) Hamiltonian $\mathscr{H}_{\text {LGW }}$ for the polymer solution to be of the form

$$
\begin{aligned}
\mathscr{H}_{\text {LGW }}= & \int d^{d} x\left\{a_{1} \varphi+\frac{1}{2} a_{2} \varphi^{2}+\frac{1}{3} a_{3} \varphi^{3}\right. \\
& \left.+\frac{1}{4} a_{4} \varphi^{4}+\frac{1}{2} b(\nabla \varphi)^{2}+\cdots\right\},
\end{aligned}
$$

with the field $\varphi$ related to the order parameter of the phase transition. While the Flory-Huggins theory does, of course, yield such an expansion in the neighborhood of the critical point, its phenomenological character precludes a systematic study of the role of fluctuations. On the other hand, the well-studied Edwards ${ }^{8}$ Hamiltonian for polymer solutions provides a representation that is expected to be more appropriate for the study of long-wavelength polymer properties near a critical point. Yet, the connection between this continuum model and the field-theoretic approaches to critical phenomena has remained incomplete. ${ }^{9}$ In the present paper we investigate this relationship.

The physical considerations and mathematical transformations that relate a microscopically defined Hamiltonian to one such as Eq. (1.8) were first discussed formally by Hubbard and Schofield ${ }^{10}$ in the context of simple fluids and are reviewed in Sec. II. A transformation of this kind has been studied in considerable detail in recent work ${ }^{11}$ on the correspondence between fluids and magnets in the critical region. Central to these results and those presented here is the basic relationship between the grand partition function $\Xi(T, \mu)$ of the fully interacting system at temperature $T$ and chemical potential $\mu$, and that of an arbitrary reference system at temperature $T$ and chemical potential $\mu_{0}$,

$$
\begin{aligned}
\Xi(T, \mu)= & \Xi_{0}\left(T, \mu_{0}\right) \\
& \times\left\langle\exp \left[-\beta\left(\mathscr{H}-\mathscr{H}_{0}\right)+\beta\left(\mu-\mu_{0}\right) n\right]\right\rangle_{0},
\end{aligned}
$$

where $n$ is the number of particles. This is the grand canonical version of the basic result used by $Z_{\text {wanzig }}{ }^{12}$ in developing high-temperature perturbation theory in the canonical ensemble. For simple fluids with a typical intermolecular potential $\phi(r)$, it is natural to partition $\phi$ into a short-range repulsive interaction, $\phi_{0}$, and a long-range attractive tail, $\phi_{1}$, such that the difference $\mathscr{H}-\mathscr{H}_{0}$ in Eq. (1.9) is simply

$$
\frac{1}{2} \sum_{i \neq j} \phi_{1}\left(r_{i j}\right)
$$

This partitioning is essentially that suggested by Weeks, Chandler, and Andersen, ${ }^{13}$ and is important for both physical and mathematical reasons. When written in Fourier space, (1.10) has the form

$$
\frac{1}{2} \sum_{\mathbf{k}} \hat{\rho}_{\mathbf{k}} \hat{\rho}_{-\mathbf{k}} \hat{\phi}_{1}(\mathbf{k}),
$$

where $\hat{\rho}_{\mathbf{k}}$ and $\hat{\phi}_{1}(\mathbf{k})$ are the Fourier transforms, respectively, of the density and the attractive potential. Since Eq. (1.11) is a quadratic form in the density variables $\hat{\rho}_{\mathbf{k}}$, a Hubbard-Stratonovich transformation may be employed to rewrite the Boltzman factor in Eq. (1.9) in terms of continuous variables. Such a Gaussian transformation requires that $-\hat{\phi}_{1}(\mathbf{k})$ be positive definite, a requirement that is well satisfied in simple fluids by the choice of a hard-sphere refer- 
ence fluid and an attractive potential $\phi_{1}$ with some smooth cutoff at short length scales.

In the Edwards model of polymer solutions, actual pair interactions are replaced by delta-function pseudopotentials, thereby preventing a clear partitioning of the overall intermolecular potential into attractive and repulsive components. In theories of the region below the theta point, where the two-body excluded-volume parameter is negative, it has proved useful to incorporate into the model repulsive three-body interactions to prevent the collapse of the system. ${ }^{14,15}$ In the present context, a natural partitioning of the Edwards Hamiltonian is one in which these three-body repulsive interactions are associated with the reference Hamiltonian, with the perturbing Hamiltonian $\mathscr{H}_{1}$ containing the attractive two-body terms. This is equivalent to choosing as the reference system the polymer solution at the theta temperature for infinite chains. ${ }^{16}$ The usefulness of this formalism rests on the ability to compute properties of this nonideal reference system. We show in Sec. II that in three dimensions such properties may be computed perturbatively around the properties of a Gaussian system. The resulting perturbation series, in which the amplitude of the three-body interaction constitutes the expansion parameter, is shown to be rapidly convergent for long chains. We emphasize that contrary to most previous approaches the present formalism requires the use of the grand canonical ensemble. This, of course, is the preferred ensemble with which to discuss fluctuations.

The perturbative incorporation of three-body interactions into the reference system for polymer solutions finds a parallel in recent studies of the role of Axilrod-Teller interactions in simple fluids. ${ }^{11,17}$ There it was found that the effects of such ternary potentials on the correlation functions of the hard-sphere reference system, computed to lowest order in a virial expansion, lead naturally to the presence of a new energy scale at the critical point, one which appears to explain certain systematic trends in nonuniversal critical amplitudes.

Our central result is the explicit form of the operators of the LGW Hamiltonian in the limit of small order parameter inhomogeneities, including the dependence of those operators on the amplitude of the ternary interaction and on the polymer molecular weight. Under the single assumption that the second osmotic virial coefficient is an analytic function of temperature near the theta point, we find that a mean field approximation to the functional integral yields a description of the bulk and interfacial critical properties that is esentially identical to that of the Flory-Huggins approximation.

In Sec. III we discuss some of the considerations that bear on generalizations of the present approach to the treatment of deviations from mean field theory.

\section{THEORY}

\section{A. General formalism}

In this section we outline the general principles underlying the transformation of the partition function of a fluid, written as a trace over particle positions, to one involving continuous fields. Having partitioned the interparticle potential $\phi(r)$ into its attractive and repulsive components, we write

$$
\mathscr{H}-\mathscr{H}_{0}=\frac{1}{2 V} \sum_{\mathbf{k}} \hat{\rho}_{\mathbf{k}} \hat{\rho}_{-\mathbf{k}} \hat{\phi}_{1}(\mathbf{k})-\frac{1}{2} n \phi_{1}(0) .
$$

We define the singular part $\Xi_{s}$ of the grand canonical partition function through the relation $\Xi=\Xi_{0} \Xi_{s}$, and introduce the reduced variables

$$
\tilde{\mu} \equiv \beta\left[\mu-\mu_{0}+\frac{1}{2} \phi_{1}(0)\right], \quad \hat{v}_{1}(\mathbf{k}) \equiv-\beta \hat{\phi}_{1}(\mathbf{k}),
$$

with $\hat{v}_{1}(\mathbf{k})>0$. Since the number of particles $n$ is just $\hat{\rho}_{0}$, we may write

$$
\Xi_{s}(T, \mu)=\left\langle\exp \left\{\tilde{\mu} \hat{\rho}_{0}+\frac{1}{2 V} \sum_{\mathbf{k}} \hat{\rho}_{\mathbf{k}} \hat{\rho}_{-\mathbf{k}} \hat{v}_{1}(\mathbf{k})\right\}\right)_{\circ} .
$$

The transformation to continuous fields is now made by recognizing that the term within curly brackets in Eq. (2.3) is a general quadratic expression, so that the identity

$$
\begin{aligned}
& \exp \left(\frac{1}{2} a^{2} x^{2}\right) \\
& \quad=\left(2 \pi a^{2}\right)^{-1 / 2} \int_{-\infty}^{\infty} d y \exp \left(-\frac{1}{2} \frac{y^{2}}{a^{2}}+x y\right)
\end{aligned}
$$

may be applied. The result is

$$
\begin{aligned}
\Xi_{s} \propto & \int \mathscr{D}[\varphi] \exp \left\{\frac{\tilde{\mu} \varphi_{0}}{\hat{v}_{1}(0)}-\frac{1}{2 V} \sum_{\mathbf{k}} \frac{1}{\hat{v}_{1}(\mathbf{k})} \varphi_{\mathbf{k}} \varphi_{-\mathbf{k}}\right\} \\
& \times\left(\exp \left(\frac{1}{V} \sum_{\mathbf{k}} \varphi_{\mathbf{k}} \hat{\rho}(-\mathbf{k})\right)\right\rangle_{0},
\end{aligned}
$$

where $\mathscr{D}[\varphi]=\Pi d \varphi_{\mathbf{k}}$, and we have suppressed analytic prefactors in the transformation which are irrelevant to the singular part of the free energy. It is the appearance of $\hat{v}_{1}(\mathbf{k})^{-1}$ in Eq. (2.5) that requires a sufficiently smooth short-distance cutoff of the attractive part of the pair potential for its Fourier transform $\hat{\phi}_{1}(\mathbf{k})$ to be negative definite. Appealing now to the cumulant theorem, ${ }^{18}$ the expectation on the righthand side of Eq. (2.5) may be written as

$$
\begin{aligned}
&\left\langle\exp \left(\frac{1}{V} \sum_{\mathbf{k}} \varphi_{\mathbf{k}} \hat{\rho}(-\mathbf{k})\right)\right\rangle_{0} \\
&=\exp \left\{\sum_{n=1}^{\infty} \frac{1}{n !} \frac{1}{V^{n}} \sum_{\mathbf{k}_{1}} \cdots \sum_{\mathbf{k}_{n}}\right. \\
&\left.\times \widehat{F}_{n}^{0}\left(\mathbf{k}_{1}, \ldots, \mathbf{k}_{n}\right) \varphi_{-\mathbf{k}_{1}} \cdots \varphi_{-\mathbf{k}_{n}}\right\}
\end{aligned}
$$

where

$$
\widehat{F}_{n}^{0}\left(\mathbf{k}_{1}, \ldots, \mathbf{k}_{n}\right)=\left\langle\hat{\rho}_{\mathbf{k}_{1}} \cdots \hat{\rho}_{\mathbf{k}_{n}}\right\rangle_{0, c}
$$

is a cumulant average in the reference system. Translational invariance implies that

$$
\widehat{F}_{n}^{0}\left(\mathbf{k}_{1}, \ldots, \mathbf{k}_{n}\right)=V \delta_{\mathbf{k}_{1}}+\cdots+\mathbf{k}_{t}, 0 \widehat{G}_{n}^{o}\left(\mathbf{k}_{1}, \ldots, \mathbf{k}_{n}\right),
$$

which serves as a definition of the $\widehat{G}_{n}^{o}$. The $\widehat{F}_{n}^{o}$ are the Fourier transforms of functional derivatives of the reference partition function, ${ }^{19}$

$$
F_{n}^{0}\left(\mathbf{r}_{1}, \ldots, \mathbf{r}_{n}\right)=\frac{\delta^{n} \ln \Xi_{0}}{\delta z\left(\mathbf{r}_{1}\right) \cdots \delta z\left(\mathbf{r}_{n}\right)},
$$

with $z(\mathbf{r})$ the spatially varying fugacity. At zero momentum, Eq. (2.9) implies the simple relation 


$$
\widehat{G}_{n}^{0}(\mathbf{0}, \ldots, 0)=\frac{1}{V} \frac{\partial^{n} \ln \Xi_{0}}{\partial\left(\beta \mu_{0}\right)^{n}},
$$

so the $\widehat{G}_{n}^{0}$ obey the recursion relation $\partial \widehat{G}_{n}^{0}(\mathbf{0}, . ., 0) / \partial\left(\beta \mu_{0}\right)$ $=\widehat{G}_{n+1}^{0}(\mathbf{0}, \ldots, 0)$. The first few cumulants are simply $\widehat{G}_{1}^{0}(\mathbf{0})=\left\langle\rho_{0}\right\rangle_{0}$, the average number density of particles in the reference system, $\widehat{G}_{2}^{0}(\mathbf{0}, 0)=\left\langle\rho_{0}^{2}\right\rangle_{0}-\left\langle\rho_{0}\right\rangle_{0}^{2}$, the variance in the reference number density, and so on.

From these results, we conclude that the singular contribution to the grand partition function is given by the integral in Eq. (1.7), where, in momentum space, the LGW Hamiltonian has a more general form than in Eq. (1.8), namely

$$
\begin{aligned}
\mathscr{H}_{\mathbf{L G W}}= & \sum_{n=1}^{\infty} \frac{1}{n !} \frac{1}{V^{n-1}} \sum_{\mathbf{k}_{1}} \cdots \sum_{\mathbf{k}_{n-1}} \\
& \times \hat{K}_{n}\left(\mathbf{k}_{1}, \ldots, \mathbf{k}_{n-1},-\mathbf{k}_{1} \cdots-\mathbf{k}_{n-1}\right) \\
& \times \varphi_{-\mathbf{k}}, \cdots \varphi_{-\mathbf{k}_{n-1}}, \varphi_{\mathbf{k}_{1}+\cdots+\mathbf{k}_{n-1}},
\end{aligned}
$$

where the operators are

$$
\begin{aligned}
& \widehat{K}_{1}(\mathbf{0})=-\widehat{G}_{1}^{o}(\mathbf{0})-\frac{\tilde{\mu}}{\hat{v}_{1}(\mathbf{0})}, \\
& \widehat{K}_{2}(\mathbf{k},-\mathbf{k})=-\widehat{G}_{2}^{o}(\mathbf{k},-\mathbf{k})+\frac{1}{\hat{v}_{1}(\mathbf{k})},
\end{aligned}
$$

and

$$
\begin{aligned}
& \hat{K}_{n}\left(\mathbf{k}_{1}, \ldots, \mathbf{k}_{n-1},-\mathbf{k}_{1}-\ldots-\mathbf{k}_{n-1}\right) \\
& \quad=-\widehat{G}_{n}^{0}\left(\mathbf{k}_{1}, \ldots, \mathbf{k}_{n-1},-\mathbf{k}_{1}-\ldots-\mathbf{k}_{n-1}\right),
\end{aligned}
$$

for all $n \geqslant 3$. Thus, given any approximation for the partition function $\Xi_{0}$ of the reference system and knowledge of the integrated strength of the attractive part of the pair potential, all of the operators of the LGW Hamiltonian may be computed.

\section{B. Application to polymer solutions}

The extension of the above ideas to a system of polymers employs a model in which the polymers are described as continuous curves in $d$ dimensions. A vector $\mathbf{r}(\tau)$ specifies the spatial location of a given segment that is an arc-length $\tau$ from the chain end. To simplify notation, we employ a system of units in which the length $\mathbf{r}=(d / l)^{1 / 2} c$, where $c$ specifies the Euclidean coordinates of the chain, and $l$ is the Kuhn length. Thus, $[\mathbf{r}]=l^{1 / 2}$. The chains are assumed to interact through two and three-body delta-function excluded volume potentials, with the total Hamiltonian $\mathscr{H}$, in units of the thermal energy $k_{B} T$, given by

$$
\begin{aligned}
\mathscr{H}= & \frac{1}{2} \sum_{i=1}^{n} \int_{0}^{N} d \tau\left|\dot{\mathbf{r}}_{i}(\tau)\right|^{2}+\frac{1}{2 !} v \sum_{i, j} \int_{0}^{N} d \tau \\
& \times \int_{0}^{N} d \tau^{\prime} \delta\left[\mathbf{r}_{i}(\tau)-\mathbf{r}_{j}\left(\tau^{\prime}\right)\right] \\
& +\frac{1}{3 !} w \sum_{i, j, k} \int_{0}^{N} d \tau \int_{0}^{N} d \tau^{\prime} \int_{0}^{N} d \tau^{\prime \prime} \\
& \times \delta\left[\mathbf{r}_{i}(\tau)-\mathbf{r}_{j}\left(\tau^{\prime}\right)\right] \delta\left[\mathbf{r}_{j}\left(\tau^{\prime}\right)-\mathbf{r}_{k}\left(\tau^{\prime \prime}\right)\right] \\
\equiv & \mathscr{H}_{g}+\mathscr{H}_{2}+\mathscr{H}_{3},
\end{aligned}
$$

where the first term $\mathscr{H}_{g}$ describes the Gaussian character of the chains in the absence of excluded-volume interactions. The phenomenological two-body excluded-volume param- eter $v$ represents the integrated strength of some potential of mean force, and is assumed to have a linear temperature dependence of the following form

$$
v(T)=v^{\prime} \frac{T-T_{\theta}}{T},
$$

with $T_{\theta}$ the theta temperature and $v^{\prime}$ some dimensionless proportionality constant. The observed positivity ${ }^{20}$ of the third virial coefficient at the theta point indicates that $w$ is positive, and this in turn is known ${ }^{21}$ to ensure the stability of the polymer against collapse below $T_{\theta}$. The actual magnitude of $w$ in real polymer solutions is somewhat uncertain, but appears to be of order $0.1-1$ as judged from the size of the third virial coefficient and the measured dimensions of polymers at the theta point. ${ }^{22,23}$

As alluded to earlier, the reference Hamiltonian $\mathscr{H}_{0}$ is taken to be $\mathscr{H}_{g}+\mathscr{H}_{3}$, with $\mathscr{H}_{2}$ acting as a perturbation. Below the theta point, this partitioning of the full Hamiltonian implies that the attractive part of the two-body potential between monomers, analogous to that in simple fluids, is

$$
\phi_{1}(\mathbf{r})=k_{B} T v \delta(\mathbf{r}), \quad \hat{v}_{1}(\mathbf{k})=v^{\prime} b\left(T_{\theta}-T\right) / T
$$

with $b$ a microscopic volume whose presence arises from the rescaled units adopted in Eq. (2.15).

For a monodisperse distribution of chain lengths, the full reference partition function then takes the form

$$
\Xi_{0}=\sum_{n=0}^{\infty} \frac{1}{n !}\left(\mathrm{q}(T) e^{\beta \mu_{i}}\right)^{n} \int \prod_{i=1}^{n} \mathscr{D}\left[r_{i}\right] e^{-*_{i}\left[r_{i}\right]},
$$

where $q(T)$ is some arbitrary function of temperature alone with the dimensions of inverse volume, which in the present system of units implies $[q]=l^{-d / 2}$. Note that $\mu_{0}$ is the chemical potential conjugate to the number of chains. The generalization of the results below to a polydisperse system is developed in Sec. II D.

In the dilute regime of interest for the critical point, where the chains are at the overlap concentration but the actual monomer concentration is low, the volume fraction of polymer $\phi \equiv n N a^{3} / V \ll 1$, with $a^{3}$ an $N$-independent elementary segment volume, may be related to the monomer density $\rho(\mathbf{r})$,

$$
\rho(\mathbf{r})=\frac{1}{l} \sum_{i=1}^{n} \int_{0}^{N} d \tau \delta\left[\mathbf{r}-\mathbf{r}_{i}(\tau)\right]
$$

by

$$
\phi=\frac{\sigma^{3}}{V} \int d^{d} r \rho(\mathbf{r}),
$$

where $\sigma^{3} / l=a^{3}$. The appearance of the Kuhn length in the definition of $\rho$ is again a consequence of the present system of units. With this definition, the reference Hamiltonian $\mathscr{H}_{0}=\mathscr{H}_{\mathrm{g}}+\mathscr{H}_{3}$ may be rewritten in a more compact form,

$$
\mathscr{H}_{0}=\frac{1}{2} \sum_{i=1}^{n} \int_{0}^{N} d \tau\left|\dot{\mathbf{r}}_{i}(\tau)\right|^{2}+\frac{1}{3 !} w \int d \mathbf{r} \rho^{3}(\mathbf{r})
$$

The identity $22,23,24$

$$
\begin{aligned}
& \exp \left[-A_{3}(\{\rho\})\right] \\
& =\left.\exp \left[-A_{3}(\{\delta / \delta J\})\right] \exp \left(\int d \mathbf{r} J(\mathbf{r}) \rho(\mathbf{r})\right)\right|_{J=0},
\end{aligned}
$$


with

$$
A_{3}[\{\rho\}]=\frac{1}{3 !} w \int d \mathbf{r} \rho(\mathbf{r})^{3},
$$

enables the canonical partition function for $n$ chains to be factorized into a product of single-chain partition functions in the presence of the external field $J$,

$\Xi_{0}=\left.\sum_{n=0}^{\infty} \frac{1}{n !} z_{0}^{n} \exp \left[-A_{3}(\{\delta / \delta J\})\right] Q(N, J)^{n}\right|_{J=0}$,

where

$$
\begin{aligned}
Q(N, J)= & \int \mathscr{D}[\mathbf{r}] \exp \left\{-\frac{1}{2} \int_{0}^{N} d \tau|\mathbf{r}(\tau)|^{2}\right. \\
& \left.+\int d \mathbf{r} \int_{0}^{N} d \tau J(\mathbf{r}) \delta[\mathbf{r}-\mathbf{r}(\tau)]\right\},
\end{aligned}
$$

is the single-chain partition function, and where we have introduced the reference fugacity $z_{0}=q(T) \exp \left(\beta \mu_{0}\right)$.

The series in Eq. (2.24) may be summed exactly to give

$$
\Xi_{0}=\left.\exp \left[-A_{3}(\{\delta / \delta J\})\right] \exp \left[z_{0} Q(N, J)\right]\right|_{J=0} .
$$

If we now define the action of the operator $\exp \left(-A_{3}\right)$ on the $k$ th term in the expansion of the exponential involving $Q$ as

$$
\left\langle Q^{k}\right\rangle \equiv e^{-A_{1}} Q^{k}
$$

we may write

$$
e^{-A_{3}} \exp \left(z_{0} Q\right)=\sum_{k=0}^{\infty} \frac{\left\langle Q^{k}\right\rangle z_{0}^{k}}{k !},
$$

The computation of $\left\langle Q^{k}\right\rangle$ is carried here to first order in the three-body amplitude $w$, and is facilitated by a diagrammatic analysis in which one expands both the operator $\exp \left(-A_{3}\right)$ and the partition function $Q$ as

$$
\begin{aligned}
& \exp \left[-A_{3}(\{\delta / \delta J\})\right] \\
& \simeq 1-\frac{1}{3 !} w \int d x \frac{\delta^{3}}{\delta J(x)^{3}}+O\left(w^{2}\right) \\
& Q(N, J)=\int \mathscr{D}[\mathbf{r}] \exp \left\{-\frac{1}{2} \int_{0}^{N} d \tau|\dot{\mathbf{r}}(\tau)|^{2}\right\} \\
& \quad \times\left\{1+\int d \mathbf{r} \int_{0}^{N} d \tau J(\mathbf{r}) \delta[\mathbf{r}-\mathbf{r}(\tau)]\right. \\
& +\frac{1}{2 !} \iint d \mathbf{r} d \mathbf{r}^{\prime} \int_{0}^{N} \int_{0}^{N} d \tau d \tau^{\prime} J(\mathbf{r}) \\
& \left.\quad \times \delta[\mathbf{r}-\mathbf{r}(\tau)] J\left(\mathbf{r}^{\prime}\right) \delta\left[\mathbf{r}^{\prime}-\mathbf{r}\left(\tau^{\prime}\right)\right]+\cdots\right\} .
\end{aligned}
$$

As a consequence of one-, two-, and three-chain ternary interactions, three types of contributions to the first-order expansion of $\left\langle Q^{k}\right\rangle$ may be distinguished. The diagrammatic structure of these terms is shown in Fig. 2. The reader is referred to Appendix A for a discussion of the evaluation of such diagrams. The resulting expansion of $\left\langle Q^{k}\right\rangle$ has the form

$\left\langle Q^{k}\right\rangle=V^{k}\left\{1-w\left[k P_{1}+2\left(\begin{array}{l}k \\ 2\end{array}\right) P_{2}+\left(\begin{array}{l}k \\ 3\end{array}\right) P_{3}\right]+O\left(w^{2}\right)\right\}$,

where $V$ is the volume of the system, and the $P_{i}$ are numerical coefficients obtained from the analysis of the diagrams.
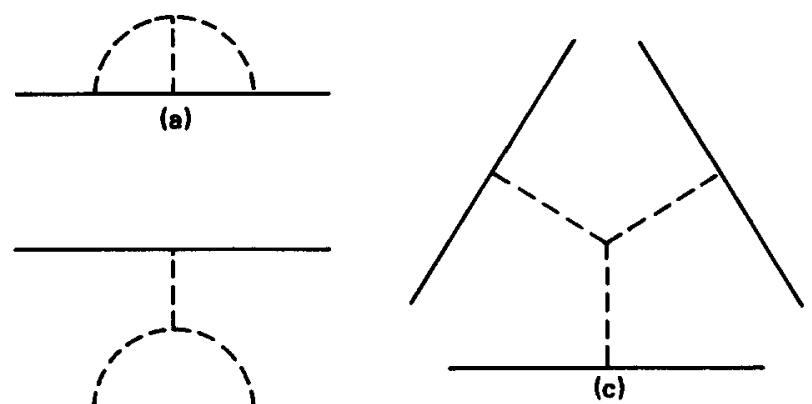

(b)

FIG. 2. Diagrammatic representation of the leading (a) one-, (b) two-, and (c) three-chain contributions to the perturbation expansion of the reference partition function in powers of the three-body excluded-volume amplitude $w$. Solid lines represent the polymer chain, dashed lines the three-body interaction.

In arriving at Eq. (2.31), we have explicitly accounted for the combinatorial factors arising from $\tau$ ordering of the segments along the chains. Summing the series in Eq. (2.28) explicitly to first order in $w$, one obtains

$\Xi_{0} \simeq e^{V z_{0}}\left\{1-w\left[z_{0} P_{1}+z_{0}^{2} P_{2}+\frac{1}{3 !} z_{0}^{3} P_{3}\right]+O\left(w^{2}\right)\right\}$

Appendix A describes the evaluation of the diagrams in Fig. 2 by the method of dimensional regularization. ${ }^{25} \mathrm{We}$ find

$$
\begin{aligned}
& P_{1}=V \frac{1}{(2 \pi)^{d}}\left(\frac{2}{d-2}\right)^{2} \frac{\Gamma(2-d / 2)^{2}}{\Gamma(4-d)} N^{3-d}, \\
& P_{2}=V \frac{1}{(2 \pi)^{d / 2}} \frac{2}{2-d} \frac{2}{4-d} N^{3-d / 2},
\end{aligned}
$$

and

$$
P_{3}=V N^{3}
$$

where $\Gamma$ is the gamma function.

Thus far, we have carried out the expansion to leading order in the three-body excluded volume amplitude $w$. To estimate the contributions from higher-order terms, consider the $n$th order diagram arising from the interaction of $3 n$ chains coupled by $n$ three-body interactions. From the pattern evident in Eqs. (2.32)-(2.35), it is apparent that the term with the strongest $N$ dependence possible at this order scales like $\left(z_{0}^{3} N^{3}\right)^{n}$. Anticipating results derived below, which estabish that the fugacities of interest at the critical point are of the order $z_{0} \sim N^{-3 / 2}$, it may be seen that in the limit of long chains that all terms beyond leading order make negligible relative contributions to the reference free energy.

From Eq. (2.32) we conclude that the logarithm of the reference partition function has the expansion

$$
\begin{aligned}
& \frac{1}{V} \ln \left(\Xi_{0}\left(T, \mu_{0}\right)\right) \\
& \quad \simeq z_{0}\left\{1-w\left[p_{1}+p_{2} z_{0} N^{3 / 2}+p_{3} z_{0}^{2} N^{3}\right]+O\left(w^{2}\right)\right\},
\end{aligned}
$$

where, in three dimensions

$$
p_{1}=1 / 2 \pi^{2}, \quad p_{2}=-4 /(2 \pi)^{3 / 2}, \quad p_{3}=1 / 6 .
$$

The cumulants $\widehat{G}_{k}^{0}$ that enter into the definition of the operators in the LGW Hamiltonian are now obtained from Eq. (2.36) by straightforward differentiation. Recall that $z_{0}$ is 
the fugacity of chains, so that the cumulants are given by $\widehat{G}_{n}^{0}(0, \ldots 0)=(1 / V) N^{n}\left[\partial^{n} \ln \Xi_{0} / \partial\left(\ln z_{0}\right)^{n}\right]$. For later use we define the reduced cumulants $g_{k}$,

$$
\begin{aligned}
g_{k}(x) & \equiv N^{-(k-3 / 2)} \hat{G}_{k}^{0}(0, \ldots, 0) \\
& =x\left\{1-w\left[p_{1}+2^{k} p_{2} x+3^{k} p_{3} x^{2}\right]+O\left(w^{2}\right)\right\},
\end{aligned}
$$

where $x \equiv N^{3 / 2} z_{0}$. These cumulants for $k=1$ to 5 are shown in Fig. 3 as a function of the fugacity variable $x$ for the case $w=0.1$. The variation of the $g_{i}$ with fugacity is of the utmost importance in obtaining a well-behaved field theory of polymer solutions. Recall that the operators $\widehat{K}_{n}$ of the LGW Hamiltonian are, for $n \geqslant 3$, simply the negative cumulants of order $n$. In a mean field theory of the functional integral which we discuss in more detail below, it is necessary that the fourth-order operator at zero momentum be positive in order that the system be stable. This requires that the corresponding fourth cumulant $\widehat{G}_{\mathbf{4}}(\mathbf{0 , 0}, \mathbf{0 , 0})$ be negative. Note that an ensemble of Gaussian chains does not have a negative fourth cumulant; indeed all cumulants are positive in the noninteracting limit. Only at sufficiently high fugacities in the presence of three-body excluded-volume interactions does the fourth cumulant of the reference system change sign. We shall see below that a natural choice of the reference fugacity $z_{0}$ is that at which the third cumulant vanishes (indicated by $x^{*}$ in Fig. 3 ), and at that point the fourth cumulant is negative, as are all higher cumulants.

It is instructive to compare the cumulant structure of the reference polymer solution with that of the analogous

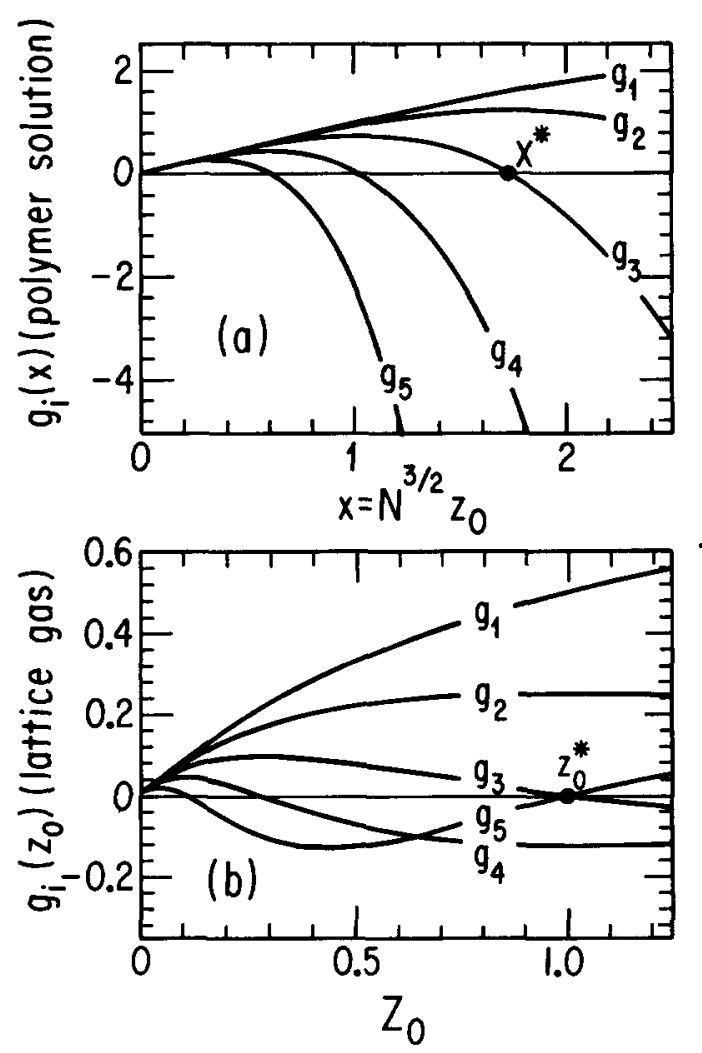

FIG. 3. Reduced cumulants of the reference polymer solution (a) and hard core lattice gas (b) as a function of fugacity. The vanishing of the third cumulant at $x^{*}$ (a) and $z_{0}^{*}$ (b) defines the mean field critical isochore of the two systems. reference system for an interacting lattice model of fluids, namely the hard core lattice gas. ${ }^{26}$ This system, in contrast to the polymer solution, exhibits an exact symmetry between the thermodynamic functions of the two phases in coexistence below the critical point. This particle-hole symmetry is perhaps most clearly seen in the structure of the cumulants. The grand partition function of this reference system is trivial, since each site can be in either of two states, empty or occupied, independent of the states of all other sites. Then,

$$
\Xi_{\mathrm{LG}}(T, \mu)=(1+z)^{\Omega}
$$

with $\Omega$ the number of lattice sites, playing the role of the volume of the system in a continuum fluid. One finds that the cumulants at zero momentum are simply

$$
\begin{aligned}
g_{n}\left(z_{0}\right) & \equiv G_{n}(0, \ldots, 0) \\
& =\frac{1}{2} \delta_{n, 1}+\frac{1}{2} \frac{\partial^{n-1} \tanh \left(\beta \mu_{0} / 2\right)}{\partial\left(\beta \mu_{0}\right)^{n-1}} .
\end{aligned}
$$

At low fugacities where the excluded-volume effects are negligible, all cumulants are positive, as in the polymer reference system, and as the density is increased the cumulants tend back toward zero, with all odd cumulants vanishing at $z_{0}=z_{0}^{*}=1$. This point corresponds to the density $\rho_{0}=1 / 2$, which is the critical isochore of the interacting lattice gas and serves as the natural density at which to choose the reference system. The fourth cumulant is negative at $z_{0}^{*}$, as in the polymer solution, and successive even cumulants oscillate in sign. Figure 3(b) illustrates the first five such cumulants. The presence in the continuous-field Hamiltonian of the polymer solution of odd terms of every order is directly related to the extreme asymmetry of the two branches of the coexistence curve, whereas the vanishing of all odd cumulants in the lattice gas reflects the underlying particle-hole symmetry of the Hamiltonian.

\section{Bulk properties in mean field theory}

The present section considers the application of the above formalism to the computation of the bulk coexistence properties of solutions of long-chain polymers within a mean field treatment. The simplest such approximation to the singular part of the Gibbs free energy neglects the momentum dependence of all operators in the LGW Hamiltonian, and identifies the free energy density with the Hamiltonian, the equilibrium value of the field $\varphi$ being that which extremizes the free energy. In keeping with the known Ising-like behavior at the critical point, the expansion is truncated at fourth order in the fields $\varphi_{\mathrm{k}}$. Converting the resulting Hamiltonian in Eq. (2.11) to real space by means of relations of the form $(1 / V) \Sigma_{k} \varphi_{k} \varphi_{-k}=\int d^{3} \mathbf{r} \varphi(\mathbf{r})^{2}$, etc., yields

$$
\begin{aligned}
& -\ln \Xi_{s} \simeq \int d^{3} r g_{s}[T, \mu ; \varphi(\mathbf{r})] \\
& =\int d^{3} r\left\{\widehat{K}_{1}(\mathbf{0}) \varphi(\mathbf{r})+\frac{1}{2 !} \widehat{K}_{2}(\mathbf{0 , 0}) \varphi(\mathbf{r})^{2}\right. \\
& +\frac{1}{3 !} \hat{K}_{3}(\mathbf{0 , 0 , 0}) \varphi(r)^{3} \\
& +\frac{1}{4 !} \widehat{K}_{4}\left(\mathbf{0 , 0 , 0 , 0 )} \varphi(r)^{4}+\cdots\right\} \text {. }
\end{aligned}
$$


Before proceeding to analyze this mean field theory, it is useful to rescale the continuous field $\varphi$. Note that the singular variation of the density of chains $\rho$ is obtained by differentiation of $\Xi_{s}$, and in conjunction with the definition of the operator $\hat{K}_{1}$ in $\mathrm{Eq} .(2.12)$, one finds

$$
\frac{1}{V} \frac{\partial \ln \Xi_{s}}{\partial \tilde{\mu}}=\rho-\rho_{0}=\frac{\langle\varphi(\mathbf{r})\rangle}{N \tilde{v}_{1}(0)} .
$$

Recalling that $\hat{v}_{1}(0) \propto T_{\theta}-T$, and anticipating the subsequent result $T_{\theta}-T_{\mathrm{c}} \sim N^{-1 / 2}$, we introduce the rescaled continuous field $\psi(\mathbf{r}) \equiv N^{1 / 2} \varphi(\mathbf{r})$, with

$$
\phi-\phi_{c}=\frac{a^{3}}{b v^{\prime}} \frac{T_{\theta}}{T_{\theta}-T_{c}} \frac{\langle\psi\rangle}{N^{1 / 2}} .
$$

The free energy density then has the expansion

$$
\begin{aligned}
& g_{s}(T, \mu)[\psi] \simeq a_{1}\left(\mu, \mu_{0}, T\right) \psi+\frac{1}{2} a_{2}\left(\mu_{0}, T\right) \psi^{2} \\
&+\frac{1}{3} a_{3}\left(\mu_{0}, T\right) \psi^{3}+\frac{1}{4} a_{4}\left(\mu_{0}, T\right) \psi^{4}+\cdots \\
& a_{n}=\frac{1}{(n-1) !} N^{-n / 2} \hat{K}_{n}(\mathbf{0}, \ldots, 0)
\end{aligned}
$$

In order that there be coexistence between two phases with differing equilibrium values of the order parameter $\varphi$ the Gibbs free energy density must exhibit two minima of equal depth. This may be achieved by requiring that both the linear and cubic terms $a_{1}$ and $a_{3}$ in the Landau expansion vanish. The vanishing of $a_{1}$ (or, equivalently, of $K_{1}$ ) leads, through Eq. (2.12), to a relation between the bare chemical potential $\mu$ and the as yet unspecified reference chemical potential $\mu_{0}$. The further vanishing of $a_{3}$ (equivalently, $K_{3}$ ) defines a locus of points $\mu_{0}^{*}(T)$ along which the truncated Landau model is symmetric, and hence is the coexistence curve in the $\mu_{0}-T$ plane. The critical point is then defined as that temperature $T_{c}$ for which $a_{2}\left(\mu_{0}^{*}\left(T_{c}\right), T_{c_{c}}\right)=0$.

The requirement that the third cumulant $\widehat{G}_{3}$ vanish to first order in $w$ is equivalent to the solution $x^{*}(w)$ of the quadratic equation

$$
27 p_{3} w\left(x^{*}\right)^{2}+8 p_{2} w x^{*}+p_{1} w-1=0,
$$

which is shown in Fig. 4. Using the relationship between the fugacity and the volume fraction of polymer in the reference system,

$$
\phi_{0}=\phi_{c}=a^{3} \widehat{G}_{1}(0)=a^{3} N^{-1 / 2} g_{1}(x),
$$

and the fact that $x^{*}$ is of order $N^{0}$, we conclude that the critical isochore in mean field theory lies at a volume fraction $\phi_{c} \simeq a^{3} N^{-1 / 2} g_{1}\left(x^{*}\right) \sim N^{-1 / 2}$, with a molecular weight dependence identical to the Flory-Huggins result, that is, $\zeta_{\phi}$ $=1 / 2$.

Further correspondence with the Flory-Huggins theory may be established by computing the deviation of the critical temperature from the theta point. Returning to the explicit form of the quadratic Landau coefficient in Eq. (2.13) and specializing to the mean field critical isochore in the neighborhood of the theta point, we deduce that

$$
a_{2}\left(T, \mu_{0}^{*}\right) \simeq \frac{1}{N}\left\{\frac{T_{\theta}}{b v^{\prime}\left(T_{\theta}-T\right)}-N^{1 / 2} g_{2}\left(x^{*}\right)\right\} .
$$

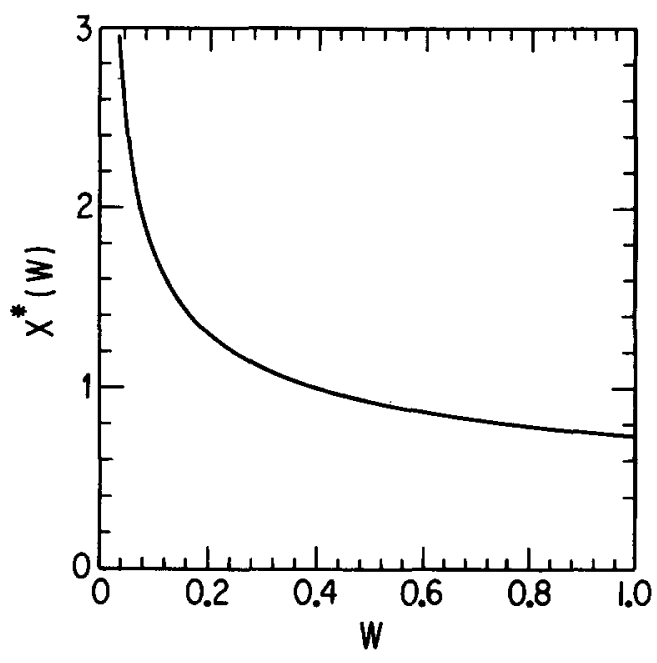

FIG. 4. Scaled fugacity $x^{*}=N^{3 / 2} z_{0}^{*}$ at which the third cumulant $\widehat{G}_{3}(\mathbf{0}, 0,0)$ vanishes, as a function of the three-body amplitude $w$.

The critical temperature thus satisfies (see Fig. 1)

$$
\Delta T \equiv T_{\theta}-T_{c} \simeq N^{-1 / 2} \frac{T_{\theta}}{b v^{\prime} g_{2}\left(x^{*}\right)},
$$

which is again the Flory-Huggins result $\xi_{T}=1 / 2$. This scaling implies, through Eq. (2.43), that $\phi-\phi_{c}$ $=\sigma^{3} g_{2}\left(x^{*}\right)\langle\psi\rangle$. Equations (2.48) and (2.49) imply that in the neighborhood of the critical point, and for $T<T_{c}$, the mean field free energy density is

$$
\begin{aligned}
g_{s}[\psi] \simeq & -\frac{1}{2}\left[b v^{\prime} g_{2}\left(x^{*}\right)^{2}\right] t \psi^{2} \\
& +\frac{1}{4}\left[-\frac{1}{6} g_{4}\left(x^{*}\right)\right] N^{1 / 2} \psi^{4}+\cdots
\end{aligned}
$$

The equilibrium order parameter follows from minimizing Eq. (2.50) as

$$
\langle\psi\rangle^{\prime \prime}-\langle\psi\rangle^{\prime}=2\left(\frac{-a_{2}\left(T, \mu_{0}^{*}\right)}{a_{4}\left(T, \mu_{0}^{*}\right)}\right)^{1 / 2} \sim N^{-1 / 4} t^{1 / 2},
$$

with $\zeta_{\beta}=1 / 4$ as in Flory-Huggins theory.

The dependence of amplitudes such as that in Eq. (2.51) on the microscopic length scales $b$ and $\sigma$ may be eliminated if the thermal scaling field $t$ is replaced by $\tau$, where

$$
\tau=\frac{T_{c}-T}{T_{\theta}-T_{c}} \simeq t b v^{\prime} g_{2}\left(x^{*}\right) N^{1 / 2},
$$

to leading order in $N$ on the mean field coexistence surface. From Eqs. (2.51) and (2.47) we find

$$
\frac{\phi^{\prime \prime}-\phi^{\prime}}{\phi_{c}}=\widetilde{A}_{1 / 2} \tau^{1 / 2} \text {. }
$$

where the $N$ independent amplitude is

$$
\widetilde{A}_{1 / 2}(w)=\sqrt{24} \frac{\left[g_{2}\left(x^{*}\right)\right]^{3 / 2}}{g_{1}\left(x^{*}\right)\left[-g_{4}\left(x^{*}\right)\right]^{1 / 2}} .
$$

In the Flory-Huggins approximation, $\tilde{A}_{1 / 2}=\sqrt{24}$, a pure number with no additional energy scales, in contrast to Eq. (2.54) in which the parameter $w$ appears explicitly. While in the Flory-Huggins model, as in the present work, all coexis- 
tence curves are predicted to collapse onto a universal form in the reduced variables $\tau$ and $N^{1 / 2} \phi$ (and perhaps with different variables in the presence of fluctuations), we see here that a small element of deviation from a pure "law of corresponding states" 27 may be introduced by the possible system-to-system variation of the three-body amplitude $w$. That is to say, there is a family of curves in the reduced variables. On the other hand, it is possible that a relationship exists between $w$ and other energy scales in the system. For instance, it has been suggested ${ }^{15}$ that $w \propto v^{2} \propto\left(T-T_{\theta}\right)^{2}$, equivalent to the statement that the third virial coefficient is proportional to the square of the second. While this is so for the entropic part of hard-sphere virial coefficients, it is not expected to hold in general for the full virial coefficients themselves. If this assertion were true, it would imply that the characteristic size of $w$ at the critical temperature is $N$ dependent through the scaling $T_{\theta}-T_{c} \sim N^{-1 / 2}$, with $w=0$ at $T_{\theta}$. The experimental observation ${ }^{20}$ of a finite third virial coefficient at the theta point of polymer solutions (where the second virial coefficient vanishes) requires that $w$ be nonzero there. We return to the question of the size of triplet interaction in Sec. III.

\section{Polydisperse systems}

Experimental studies of phase equilibrium in polymer solutions are generally carried out on polydisperse samples, and it is, therefore, of interest to generalize the results obtained above to systems with a distribution of chain lengths. For this purpose, we extend the definition of monomer density in Eq. (2.19) to include a sum over the different polymer species present, there being $n_{k}$ polymers of length $N_{k}$,

$$
\rho(\mathbf{r})=\frac{1}{l} \sum_{k} \sum_{i=1}^{n_{k}} \int_{0}^{N_{k}} d \tau \delta\left[\mathbf{r}-\mathbf{r}_{k l}(\tau)\right] .
$$

The reference partition function is now given by

$$
\begin{aligned}
\Xi_{0}= & \prod_{k} \sum_{n_{k}=0}^{\infty} \frac{1}{\prod n_{k} !} \prod_{k}\left(z_{0, k}\right)^{n_{k}} \\
& \times \int \prod_{k} \prod_{i=1}^{n_{k}} \mathscr{D}\left[\mathbf{r}_{i k}\right] e^{-\mathscr{E}_{\mathrm{o}}\left[\mathbf{r}_{i}\right]},
\end{aligned}
$$

where the reference Hamiltonian becomes

$$
\begin{aligned}
\mathscr{H}_{0}= & \frac{1}{2} \sum_{k} \sum_{i=1}^{n_{k}} \int_{0}^{N_{k}} d \tau\left|\dot{\mathbf{r}}_{i k}(\tau)\right|^{2} \\
& +\frac{1}{3 !} w \sum_{l, m, n} \sum_{i=1}^{n_{l}} \sum_{j=1}^{n_{m}} \sum_{k=1}^{n_{n}} \int_{0}^{N_{l}} d \tau \int_{0}^{N_{m}} d \tau^{\prime} \int_{0}^{N_{n}} d \tau^{\prime \prime} \\
& \times \delta\left[r_{i l}(\tau)-r_{j m}\left(\tau^{\prime}\right)\right] \delta\left[r_{j m}\left(\tau^{\prime}\right)-r_{k n}\left(\tau^{\prime \prime}\right)\right]
\end{aligned}
$$

while the perturbing Hamiltonian takes the form

$$
\begin{aligned}
\mathscr{H}_{1}= & \frac{1}{2 !} v \sum_{k, l} \sum_{i=1}^{n_{k}} \sum_{j=1}^{n_{l}} \int_{0}^{N_{k}} d \tau \\
& \times \int_{0}^{N_{l}} d \tau^{\prime} \delta\left[\mathbf{r}_{i k}(\tau)-\mathbf{r}_{j l}\left(\tau^{\prime}\right)\right],
\end{aligned}
$$

with $v(T)$ as in Eq. (2.16), assumed the same for all chains.

In Appendix B we show how the diagrammatic analysis described in Sec. II C of the perturbation expansion of the reference partition function is modified by the occurrence of polydispersity. In the grand canonical ensemble, polydispersity is represented by a set of distinct chemical potentials for the species $l$, and it proves convenient to define certain moments of the distribution of chain lengths in terms of the reference fugacities $z_{0, k}$ as

$$
\left\langle N^{\alpha}\right\rangle=\sum_{k} z_{0, k} N_{k}^{\alpha} / \sum_{k} z_{0, k}
$$

Defining further the total fugacity $\bar{z}_{0}$ as

$$
\bar{z}_{0} \equiv \sum_{k} z_{0, k}
$$

we find, in three dimensions, that the logarithm of the reference partition function has an expansion in the three-body amplitude with the same form as in the monodisperse case [Eq. (2.36)], namely,

$$
\begin{aligned}
\frac{1}{V} \log \Xi_{0} \simeq \bar{z}_{0}\left\{1-w\left[p_{1}+p_{2}\langle N\rangle\left\langle N^{1 / 2}\right\rangle \bar{z}_{0}\right.\right. \\
\left.\left.+p_{3}\langle N\rangle^{3} \bar{z}_{0}^{2}\right]+O\left(w^{2}\right)\right\} .
\end{aligned}
$$

The vanishing of the third cumulant of the reference system defines, as before, the mean field critical isochore. The relation between the total volume fraction and total fugacity leads, through Eq. (2.61), to the scaling result for the critical composition

$$
\phi_{c} \propto\left\{\langle N\rangle\left[1+\left(\frac{\langle N\rangle-\left\langle N^{1 / 2}\right\rangle^{2}}{\left\langle N^{1 / 2}\right\rangle^{2}}\right)\right]\right\}^{-1 / 2} .
$$

For a monodisperse distribution, Eq. (2.62) reduces to the Flory-Huggins result $\zeta_{\phi}=1 / 2$.

Note that the corrections for polydispersity involve the variance in the radius of gyration $N^{1 / 2}$ (for Gaussian chains ), in contrast to the Flory-Huggins result in which the variance in molecular weight $N$ enters. ${ }^{28}$ That the radius of gyration should be the fundamental quantity in the discussion of polydisperse properties may perhaps be understood as arising from the resemblance of the polymer critical point to that of simple fluids, the size of the polymer coil playing the role of the hard sphere radius.

\section{E. Interfacial properties}

The generalization of the mean field models of polymer solutions to situations in which an interface is present requires the determination of the molecular weight dependence of the nonlocal (i.e., square-gradient) contribution to the free energy. In the simplest such model, that of van der Waals, Widom ${ }^{29}$ has pointed out that the nonlocal contribution to the free energy density could be taken as independent of $N$, in the same way that the energetic component of the Flory-Huggins free energy, which originates in the statistics of unconnected segments, is independent of $N$. This approach leads to the result $\zeta_{\mu}=1 / 2$, in disagreement with experiment. It was shown, however, that a generalization of certain scaling arguments ${ }^{21}$ could be used to find the non- 
classical value of $\zeta_{\mu}$ given its classical value. The application of these ideas to the recent mean field theories of Nose and of Vrij and Roebersen, which use both the local density of chain centers and of chain segments as order parameters, leads to the nonclassical value $\zeta_{\mu} \simeq 0.37$, in reasonable agreement with experiment. This level of agreement does not appear, however, when such scaling arguments are applied to the theory with an $N$ independent nonlocal contribution. Stepanow ${ }^{30}$ has presented analogous scaling arguments for bulk exponents, finding reasonable agreement with the nonclassical observations.

The present analysis of interfacial properties makes use of the van der Waals ${ }^{31}$ theory. Here, the interface separates the two phases, one polymer rich, the other solvent rich, which coexist below the critical temperature. For this purpose, we adopt a variational approach to the spatial profile of the monomer density within a square-gradient approximation. We continue to work with the variable $\psi$, proportional to the deviation of the local volume fraction from $\phi_{c}$ with an $N$ independent constant of proportionality. The surface tension then takes the form

$$
\tilde{\sigma}=\min _{[\psi(x)]} \int_{-\infty}^{\infty} d z\left\{\frac{1}{2} m\left(\frac{d \psi}{d z}\right)^{2}+\Gamma(\psi)\right\}
$$

where the function $\Gamma(\psi)$ is the deviation of the bulk free energy density, evaluated at the local $\psi(z)$, from the common tangent to the free energy surface,

$$
\Gamma(\psi)=g_{s}[\psi(z)]-g_{s}\left(\psi_{b}\right),
$$

with $g_{s}\left(\psi_{b}\right)$ the common value of the Gibbs free energy density in the two phases.

It is first necessary to compute the coefficient of $(\nabla \psi)^{2}$ in Eq. (2.63), and from the operator structure of the LGW Hamiltonian defined in Eqs. (2.11)-(2.14), this is equivalent to the determination of the coefficient of (momentum) ${ }^{2}$ in the small-momentum expansion of the operator $\widehat{K}_{2}(\mathbf{k},-\mathbf{k})$. We see that contributions to $m$ arise both from the potential $\phi_{1}$ and from the reference system correlation function $G_{2}$. This is in contrast to the original formulation of van der Waals, in which the coefficient $m$ of the gradientsquared term in Eq. (2.63) is purely energetic, being proportional to the square of the range of the pair potential through which particles interact. More rigorous formulations ${ }^{31}$ show it to be governed by the second moment of the two-particle direct correlation function of the homogeneous fluid, and the quantity $\widehat{K}_{2}$ is essentially a mean spherical form of that function. The momentum independence of the Fourier transform of the delta function pseudopotential between chain segments implies that the only contribution to this expansion comes from the reference cumulant $\widehat{G}_{2}^{0}(\mathbf{k},-\mathbf{k})$, the computation of which is provided below. Thus, if one considers the polymer reference system as analogous to the purely entropic hard-core reference fluid used to describe systems of small molecules, then the nonlocal contribution to the free energy is likewise "entropic" in origin. That it should depend on molecular weight is then clear.

In general, $\widehat{G}_{2}^{o}(\mathbf{k},-\mathbf{k})=\left\langle\hat{\rho}_{\mathbf{k}} \hat{\rho}_{-\mathbf{k}}\right\rangle_{0}-\left\langle\hat{\rho}_{\mathbf{k}}\right\rangle_{0}\left\langle\hat{\rho}_{-\mathbf{k}}\right\rangle_{0}$, but, from the nature of cumulant expansions, only the connected diagrams which enter into the quantity

$$
\begin{aligned}
\left\langle\hat{\rho}_{\mathbf{k}} \hat{\rho}_{-\mathbf{k}}\right\rangle_{0}= & \sum_{j, k} \int d \mathbf{r} \int d \mathbf{r}^{\prime} \int_{0}^{N} d s \int_{0}^{N} d s^{\prime} \\
& \times e^{-i \mathbf{k}\left(\mathbf{r}-\mathbf{r}^{\prime}\right)}\left\langle\delta\left[\mathbf{r}-\mathbf{r}_{j}(s)\right]\right. \\
& \left.\times \delta\left[\mathbf{r}^{\prime}-\mathbf{r}_{k}\left(s^{\prime}\right)\right]\right\rangle_{0}
\end{aligned}
$$

need be considered. The techniques described in Sec. II C enable this average to be represented as

$$
\begin{gathered}
\Xi_{0}^{-1} \sum_{n=0}^{\infty} \frac{z_{0}^{n}}{n !} \exp \left[-A_{3}(\{\delta / \delta J\})\right] \int \prod_{i=1}^{n} \mathscr{D}\left[\mathbf{r}_{i}\right] \\
\quad \times \sum_{j} \sum_{k} \int d \mathbf{r} \int d \mathbf{r}^{\prime} \int_{0}^{N} d s \int_{0}^{N} d s^{\prime} e^{-i \mathbf{k}\left(\mathbf{r}-\mathbf{r}^{\prime}\right)} \\
\quad \times \delta\left[\mathbf{r}-\mathbf{r}_{j}(s)\right] \delta\left[\mathbf{r}-\mathbf{r}_{k}\left(s^{\prime}\right)\right] \prod_{i=1}^{n} \\
\quad \times \exp \left\{-\frac{1}{2} \int_{0}^{N} d \tau\left|\dot{\mathbf{r}}_{i}(\tau)\right|^{2}\right. \\
\left.\quad+\int d \mathbf{r} \int_{0}^{N} d \tau J(\mathbf{r}) \delta\left[\mathbf{r}-\mathbf{r}_{i}(\tau)\right]\right\}
\end{gathered}
$$

Appendix A describes the structure of the expression in Eq. (2.66), which is represented diagrammatically in Fig. 5. We find, by dimensional regularization of the integrals appearing in the expansion, that the second cumulant has the small momentum behavior

$$
\begin{aligned}
\hat{G}_{2}^{o}(\mathbf{k},-\mathbf{k}) \simeq N^{1 / 2} g_{2}(x) \\
\quad-\frac{1}{2} N^{3 / 2} x\left\{1+w\left[r_{1}+r_{2} x+r_{3} x^{2}\right]\right. \\
\left.\quad+O\left(w^{2}\right)\right\} k^{2}+O\left(k^{4}\right)
\end{aligned}
$$

with the numerical coefficients obtained from those tabulated in Table I,

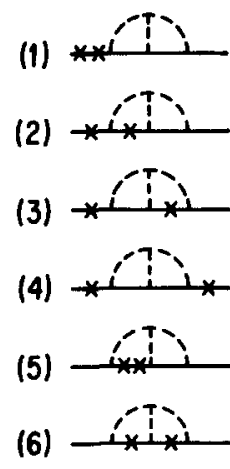

(7)

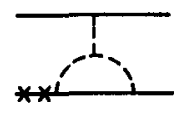

(B)
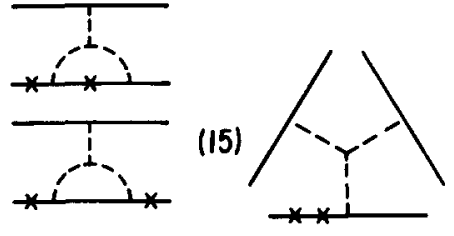

(10)

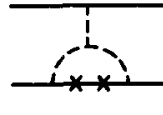

(II)

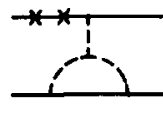

(16)

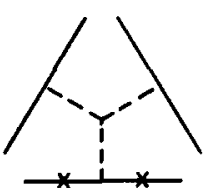

(I2)

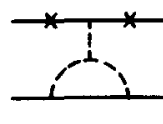

(17)

(13)
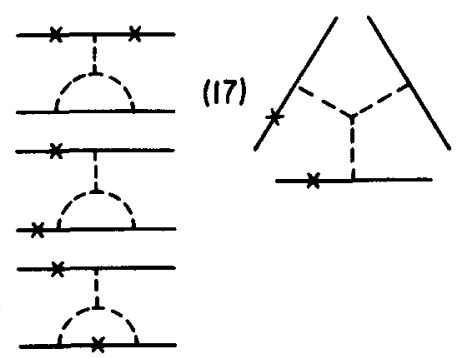

(14)

FIG. 5. Diagrammatic representation of the contributions linear in $w$ to the perturbation expansion of the second cumulant $\widehat{G}_{2}^{0}(\mathbf{k},-\mathbf{k})$. Notation is as in Fig. 2, with crosses representing the positions of the two scattering centers. Values of the diagrams are shown in Table $I$. 
TABLE I. Values of the diagrams appearing in Fig. 4, in three dimensions.

\begin{tabular}{rcc}
\hline \hline$i$ & $V^{-1} N^{-2}(2 \pi)^{3} D_{i}(0)$ & $V^{-1} N^{-3}(2 \pi)^{3} D_{;}^{\prime \prime}(0)$ \\
\hline 1 & $2 \pi$ & $-2 \pi$ \\
2 & $-\pi$ & $(13 / 12) \pi$ \\
3 & $-\pi$ & $(13 / 12) \pi$ \\
4 & $2 \pi$ & $-4 \pi$ \\
5 & $-(1 / 4) \pi$ & $-(1 / 16) \pi$ \\
6 & $(1 / 1) \pi$ & $\left.V^{-1}\right) \pi / 2(2 \pi)^{3 / 2} D_{i}^{\prime \prime}(0)$ \\
& $V^{-1} N^{-7 / 2}(2 \pi)^{3 / 2} D_{i}(0)$ & $32 / 35$ \\
7 & $-16 / 15$ & $-52 / 105$ \\
8 & $8 / 15$ & $64 / 35$ \\
9 & $-16 / 15$ & $-1 / 35$ \\
10 & $2 / 15$ & $1 / 4$ \\
11 & $-2 / 3$ & 1 \\
12 & $-2 / 3$ & $44 / 15$ \\
13 & $-4 / 3$ & $-11 / 15$ \\
14 & $2 / 3$ & $V^{-6} D_{i}^{\prime \prime}(0)$ \\
& $V^{-1} N^{-5} D_{i}(0)$ & $-1 / 8$ \\
15 & $1 / 6$ & $-1 / 4$ \\
16 & $1 / 6$ & $-1 / 2$ \\
17 & $1 / 4$ & \\
\hline \hline
\end{tabular}

$$
r_{1}=-\frac{43}{6(2 \pi)^{2}}, \quad r_{2}=\frac{605}{21(2 \pi)^{3 / 2}}, \quad r_{3}=-\frac{5}{2},
$$

in three dimensions. When evaluated on the mean field critical isochore, where $x=x^{*} \sim O\left(N^{0}\right)$, we find that the net effect of the three-body interactions has simply been to alter the numerical prefactor of the Gaussian chain behavior $N^{3 / 2} k^{2}$. From the rescaling of the Landau coefficients in Eq. (2.45), we find that the coefficient of (gradient) ${ }^{2}$ in Eq. (2.63) is of the from

$$
m\left(w, N, x^{*}\right) \simeq m_{0} N^{1 / 2},
$$

with some $N$ independent function $m_{0}(w)$. The explicit molecular weight dependence of the nonlocal part of the square gradient free energy density distinguishes the present calculation from one discussed by Widom. ${ }^{29}$

We treat here only the region asymptotically close to the critical point, and approximate $g_{s}$ by its expansion truncated at fourth order in the order parameter $\psi, g_{s}[\psi(z)]$ $\simeq(1 / 2) a_{2} \psi(z)^{2}+(1 / 4) a_{4} \psi(z)^{4}$, with $g_{s}\left[\psi_{b}\right]=-a_{2}^{2} / 4 a_{4}$. The Euler-Lagrange equation for the interfacial profile is

$$
m_{0}(w) N^{1 / 2} \frac{d^{2} \psi}{d z^{2}}=\frac{d \Gamma(\psi)}{d \psi}
$$

This functional equation displays the explicit $N$ dependence of the nonlocal contribution to the free energy. With the boundary conditions $\psi(z= \pm \infty)= \pm \psi_{b}$, Eq. (2.70) yields the standard profile

$$
\psi(z)=\psi_{b} \tanh (z / 2 \xi),
$$

where the width of the interfacial region is proportional to the correlation length

$$
\xi \equiv\left(\frac{m}{-2 a_{2}}\right)^{1 / 2} \sim^{1 / 4} t^{-1 / 2}
$$

The power $1 / 4$ of the molecular weight dependence of $\xi$ is the same as that of de Gennes'32 scaling hypothesis,

$$
\xi \sim N^{1 / 2}\left(\frac{T_{\theta}-T_{c}}{T_{c}-T}\right)^{v},
$$

with the mean field approximation to the correlation length exponent, $v=1 / 2$, and the scaling $T_{\theta}-T_{c} \sim N^{-1 / 2}$. The surface tension itself is

$$
\tilde{\sigma}=\frac{2^{3 / 2}}{3} \frac{m^{1 / 2}}{a_{4}}\left(-a_{2}\right)^{3 / 2} \sim N^{-1 / 4} t^{3 / 2},
$$

with the mean field exponent $\mu=3 / 2$. The $N$ dependence obtained here is identical to that found by Vrij and Roeber$\operatorname{sen}^{5}$ and by Nose ${ }^{6}$ who, as remarked earlier, considered two distinct spatially-varying order parameters in the description of the interfacial structure. In the present approach there is a single degree of freedom, the local monomer density $\rho(r)$ [or, equivalently, the scaled quantity $\psi(\mathbf{r})]$.

\section{DISCUSSION}

The present work establishes a systematic and controlled path from a microscopic continuum model of polymer solutions to a field-theoretic description of the critical properties of such a system. It reveals an interesting parallel between the descriptions of critical phenomena in simple fluids ${ }^{11}$ and in polymeric mixtures in which the effects of three-body interactions are incorporated perturbatively into a reference system, with the attractive interactions treated by means of a Hubbard-Stratonovich transformation to a continuous field representation. A microscopic definition of the square-gradient term in the free energy density of a weakly inhomogeneous system reveals an important entropic contribution absent in the van der Waals picture, one which is shown here to imply a functional equation for the interfacial profile with an explicit dependence on the polymer molecular weight. Interestingly, the entropic portion of the square-gradient coefficient also plays in important role ${ }^{11}$ in the validity of particle-hole symmetry in critical fluids through its density dependence. There too, three-body interactions alter the magnitude of the square-gradient coefficient.

Whereas the phase behavior of a model on the level of that due to Flory and Huggins obeys an exact law of corresponding states, ${ }^{27}$ such is not the case with the Edwards model studied here if the phenomenological three-body excluded-volume parameter $w$ is considered system dependent. On the other hand, we have found that the scaling behavior of the critical phenomena arising from the Edwards model is essentially identical to the Flory-Huggins results, which arise from a lattice model with no explicit three-body interactions at all. One is thus led naturally to inquire into the relation between the Edwards model and the original lattice description. Is the presence of a three-body term in the Edwards model a consequence of real three-body forces in the underlying lattice description, or is it instead a consequence of the nature of the continuum limit of a system with purely pairwise-additive interactions? If, as would appear to be the case, the latter is true, then one expects a relationship between the three-body amplitude $w$ and other 
energy scales in the problem. Indeed, a study ${ }^{33}$ of this issue within the context of field-theoretic representations ${ }^{34,35}$ of lattice models of interacting, self-avoiding polymers ( $n$ vector models in the limit $n \rightarrow 0^{36}$ ) suggests that the continuum limit of such lattice systems is an Edwards model with arbitrarily high-order many-body excluded-volume interactions whose strength is related in a simple way to the thermal energy at the theta point.

In the present work, we have confined the analysis to the immediate region of the consolute point, emphasizing the molecular weight dependence of the critical amplitudes defined in the asymptotic critical region $t \rightarrow 0$ at fixed polymerization index $N$. Elsewhere, ${ }^{37}$ following Widom, ${ }^{29}$ we will address in detail more general scaling properties of the phase boundary and surface tension with both molecular weight and temperature within the critical region.

Having established an exact and analytically tractable formalism for the study of critical phenomena in polymer solutions by field-theoretic techniques, it is naturally of interest to investigate fluctuation corrections to the mean field results obtained here, both at the level of Gaussian fluctuation theory ${ }^{38}$ and with more sophisticated renormalization group techniques. ${ }^{39}$ Of particular interest is the fact that the LGW model obtained here is identical in form to that of an Ising-like system, with the polymeric nature of the solute entering only through the parametric dependence on $N$ of the various operators in the Hamiltonian. Unlike the Ising model, this field-theoretical Hamiltonian contains certain symmetry-breaking odd operators which render an analysis of coexistence curve more subtle. In mean field approximation the critical isochore is defined by the vanishing of the third cumulant of the reference system. An investigation of fluctuation corrections to the mean field coexistence surface in the space of the coefficients of such asymmetric models ${ }^{40}$ may be a fruitful approach toward an understanding of the nonclassical exponent governing the molecular weight dependences of the critical composition. ${ }^{37}$ The nonclassical exponents found in critical amplitudes would follow from similar techniques applied to the scaling law equation of state.

\section{ACKNOWLEDGMENTS}

We are most grateful to Professor B. Widom for stimulating discussions leading to the work reported here and for his encouragement and insights, and to M. E. Cates, J. des Cloiseaux, B. Duplantier, K. F. Freed, L. Leibler, S. Leibler, A. Parola, and A. I. Pesci for important discussions. This work was supported in part by a graduate fellowship from the Fannie and John Hertz Foundation at Cornell University, by a Robert McCormick Postdoctoral Fellowship at the University of Chicago (R.E.G.), and by the National Science Foundation, through the Materials Research Laboratory at the University of Chicago, Grant No. DMR8519460 .

\section{APPENDIX A: CALCULATION OF THE REFERENCE PARTITION FUNCTION}

As an example of the methods used to determine the properties of the reference system, we consider in this Ap- pendix the calculation of a particular two-chain contribution to the second cumulant arising from three-body interactions. The full expression for $\widehat{G}_{2}^{0}(k,-k)$ is

$$
\begin{aligned}
\left\langle\hat{\rho}_{\mathbf{k}} \hat{\rho}_{-\mathbf{k}}\right\rangle_{0}-\left\langle\hat{\rho}_{\mathbf{k}}\right\rangle_{0}\left\langle\hat{\rho}_{-\mathbf{k}}\right\rangle_{0} & \\
= & z_{0}\left\{S_{g}(\kappa)-2 w\left[2\left(D_{1}+D_{2}+D_{3}+D_{5}\right)+D_{4}+D_{6}\right.\right. \\
& +z_{0}\left(2\left(D_{7}+D_{8}+D_{11}+D_{14}\right)\right. \\
& \left.+D_{9}+D_{10}+D_{12}+4 D_{13}\right) \\
& \left.\left.+\frac{1}{2} z_{0}^{2}\left(2 D_{15}+D_{16}+4 D_{17}\right)\right]+O\left(w^{2}\right)\right\},
\end{aligned}
$$

with $D_{n}=D_{n}(\mathbf{k})$ standing for the labelled diagrams in Fig. 5. Here, the scattering function of a Gaussian chain is

$$
S_{g}(\kappa)=2\left\{\frac{1}{\kappa}+\frac{1}{\kappa^{2}}\left(e^{-\kappa}-1\right)\right\},
$$

with $\kappa=N k^{2} / 2$, and is represented by a diagram with a single polymer chain and two scattering points. At zero momentum, the individual $D_{i}$ sum up with the weights given in Eq. (A1) to give the coefficients in Eq. (2.38); i.e., $N^{2} P_{1}=2\left[2\left(D_{1}+D_{2}+D_{3}+D_{5}\right)+D_{4}+D_{6}\right], \quad 2^{2} N^{2} P_{2}$ $=2\left[2\left(D_{7}+D_{8}+D_{11}+D_{14}\right)+D_{9}+D_{10}+D_{12}+4 D_{13}\right]$, and $3^{2} N^{2} P_{3} / 3 !=2 D_{15}+D_{16}+4 D_{17}$.

We consider the evaluation of the general polydisperse diagram $D_{14}$ in Fig. 5 (shown with labelled internal variables in Fig. A1), to illustrate the application of the method of dimensional regularization. At zero momentum, it is simpler to study the corresponding diagram $P_{2}$ shown in Fig. 2, to which $D_{14}$ contributes as described above. From the expansion of the operator $A_{3}$ in Eqs. (2.29) and (2.30), we obtain

$$
P_{2}=\int d \mathbf{x} \frac{\delta^{3}}{\delta J(\mathbf{x})^{3}} R_{2},
$$

where

$$
\begin{aligned}
R_{2}= & \int d \mathbf{R}_{l} \int d \mathbf{r}_{l} \int d \mathbf{R}_{m} \int d \mathbf{r}_{m} \\
& \times \int d \mathbf{r} \int d \mathbf{r}^{\prime} \int d \mathbf{r}^{\prime \prime} \int_{0}^{N_{l}} d \tau \int_{0}^{N_{m}} d \tau^{\prime} \int_{0}^{\tau^{\prime}} d \tau^{\prime \prime} \\
& \times G_{0}\left(\mathbf{r}-\mathbf{r}_{l}, \tau\right) J(\mathbf{r}) G_{0}\left(\mathbf{R}_{l}-\mathbf{r}, N_{l}-\tau\right) \\
& \times G_{0}\left(\mathbf{r}^{\prime}-\mathbf{r}_{m}, \tau^{\prime}\right) J\left(\mathbf{r}^{\prime}\right) G_{0}\left(\mathbf{r}^{\prime \prime}-\mathbf{r}^{\prime}, \tau^{\prime \prime}-\tau^{\prime}\right) \\
& \times J\left(\mathbf{r}^{\prime \prime}\right) G_{0}\left(R_{m}-\mathbf{r}^{\prime \prime}, N_{m}-\tau^{\prime \prime}\right)
\end{aligned}
$$

where the zero-order Green's function is

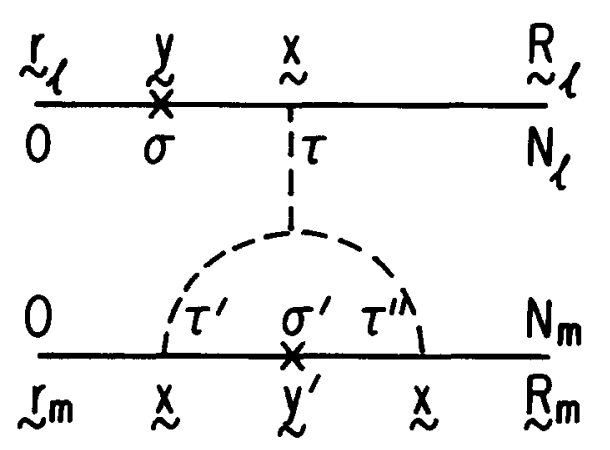

FIG. A1. Two-chain diagram (number 14 of Fig. 5) contributing to the perturbation expansion of the second cumulant, labelled as in the general polydisperse case with scattering points $\mathbf{y}$ and $\mathbf{y}^{\prime}$. 


$$
G_{0}(\mathbf{R}, N)=(2 \pi N)^{-d / 2} \exp \left(-\mathbf{R}^{2} / 2 N\right) .
$$

The integrations over the end-vectors $\mathbf{R}_{l}, \mathbf{R}_{m}, \mathbf{r}_{l}$, and $\mathbf{r}_{m}$ and the internal coordinate $\mathbf{x}$ yield unity by the normalization and translational invariance of the unperturbed Green's functions, with the result

$$
P_{2}=V \frac{1}{(2 \pi)^{d / 2}} \int_{0}^{N_{t}} d \tau \int_{0}^{N_{m}} d \tau^{\prime} \int_{0}^{\tau^{\prime}} d \tau^{\prime \prime}\left(\tau^{\prime}-\tau^{\prime \prime}\right)^{-d / 2}
$$

The $\tau$ integral trivially yields a factor of $N_{l}$, and dimensional regularization of the remaining integral (convergent for $d<2$ ) leads to the final result

$$
P_{2}=V \frac{1}{(2 \pi)^{d / 2}} \frac{2}{2-d} \frac{2}{4-d} N_{l} N_{m}^{2-d / 2} .
$$

In the monodisperse case, $N_{t}=N_{m}$, this reduces to the value quoted in Eq. (2.34).

We wish to compute

$$
\left.D_{i}^{\prime \prime}(0) \equiv \sum_{i=1}^{d} \frac{\partial^{2}}{\partial k_{i}^{2}} D_{i}(k)\right|_{k=0} .
$$

The evaluation of the diagram in Fig. A1 at finite momentum $\mathbf{k}$ is facilitated by considering its Fourier-Laplace representation. The complete expression is

$$
\begin{aligned}
D_{14}\left(N_{l}, N_{m} ; k\right) & \\
= & \int d \mathbf{y} \int d \mathbf{y}^{\prime} \int d \mathbf{R}_{l} \int d \mathbf{r}_{l} \int d \mathbf{R}_{m} \int d \mathbf{r}_{m} \\
& \times \int d \mathbf{x} \int d \sigma \int d \sigma^{\prime} \int d \tau \int d \tau^{\prime} \int d \tau^{\prime \prime} \\
& \times e^{i k \cdot\left(\mathbf{y}-\mathbf{y}^{\prime}\right)} G_{0}\left(\mathbf{y}-\mathbf{r}_{l}, \sigma\right) G_{0}(\mathbf{x}-\mathbf{y}, \tau-\sigma) \\
& \times G_{0}\left(\mathbf{R}_{l}-\mathbf{x}, N_{l}-\tau\right) \\
& \times G_{0}\left(\mathbf{x}-\mathbf{r}_{m}, \tau^{\prime}\right) G_{0}\left(\mathbf{y}^{\prime}-\mathbf{x}, \sigma^{\prime}-\tau^{\prime}\right) \\
& \times G_{0}\left(\mathbf{x}-\mathbf{y}^{\prime}, \tau^{\prime \prime}-\sigma^{\prime}\right) G_{0}\left(\mathbf{R}_{m}-\mathbf{x}, N_{m}-\tau^{\prime \prime}\right) .
\end{aligned}
$$

Introducing the double Laplace transform $\hat{D}_{14}\left(s_{l}, s_{m} ; k\right)$ $=\mathscr{L}\left[D_{14}\left(N_{l}, N_{m} ; k\right)\right]$ and using the convolution property of the transform of the time-ordered diagram, we find

$$
\begin{aligned}
\widehat{D}_{14}\left(s_{l}, s_{m} ; k\right)= & V\left[\widehat{G}_{0}\left(\mathbf{0}, s_{l}\right)\right]^{2}\left[\widehat{G}_{0}\left(\mathbf{0}, s_{m}\right)\right]^{2} \\
& \times \widehat{G}_{0}\left(-\mathbf{k}, s_{l}\right) \int \frac{d^{d} q}{(2 \pi)^{d}} \\
& \times \widehat{G}_{0}\left(\mathbf{q}, s_{m}\right) \widehat{G}_{0}\left(\mathbf{k}+\mathbf{q}, s_{m}\right),
\end{aligned}
$$

where the Fourier-Laplace transform of the zero-order Green's function (A5) is

$$
\widehat{G}_{0}(\mathbf{k}, s)=\left(s+\frac{1}{2} k^{2}\right)^{-1}
$$

To evaluate the low-momentum behavior of (A10), use the relationships ${ }^{41}$

$$
\frac{1}{a^{\alpha} b^{\beta}}=\frac{\Gamma(\alpha+\beta)}{\Gamma(\alpha) \Gamma(\beta)} \int_{0}^{1} \frac{x^{\alpha-1}(1-x)^{\beta-1}}{[a x+b(1-x)]^{\alpha+\beta}},(
$$

and

$$
\begin{aligned}
\int d^{d} p & \frac{1}{\left[p^{2}+2 \mathbf{k} \cdot \mathbf{p}+m^{2}\right]^{\alpha}} \\
& =\frac{\Gamma(\alpha-d / 2)}{\Gamma(\alpha)} \frac{\pi^{d / 2}}{\left(m^{2}-k^{2}\right)^{\alpha-d / 2}},
\end{aligned}
$$

to rewrite Eq. (A10) as

$$
\begin{aligned}
\hat{D}_{14}\left(s_{l}, s_{m} ; k\right)= & V \frac{1}{\pi} \frac{1}{s_{l}^{2}} \frac{1}{s_{m}^{2}} \frac{1}{k^{2}+2 s_{l}} \\
& \times \int_{0}^{1} d x \frac{1}{\left[2 s_{m}+x(1-x) k^{2}\right]^{1 / 2}} .
\end{aligned}
$$

Subsequent expansion for small $k$ and inverse Laplace transformation, using $\mathscr{L}^{-1}\left[s^{-a}\right]=N^{a-1} / \Gamma(a)$, gives

$$
\begin{aligned}
D_{14}\left(N_{l}, N_{m} ; k\right)= & V \frac{1}{(2 \pi)^{3 / 2}}\left\{\frac{2}{3} N_{l}^{2} N_{m}^{5 / 2}\right. \\
& -\frac{1}{90}\left(N_{l}^{2} N_{m}^{5 / 2}\right. \\
& \left.\left.+10 N_{l}^{3} N_{m}^{3 / 2}\right) k^{2}+O\left(k^{4}\right)\right\} .
\end{aligned}
$$

In the monodisperse case, this yields the $N^{7 / 2}$ dependence shown in Table I.

\section{APPENDIX B: POLYDISPERSE SYSTEMS}

In this Appendix we give further details of the generalization of the perturbation theory to polydisperse systems. The three-body interactions in the polydisperse reference Hamiltonian Eq. (2.57) may be rewritten as in the monodisperse case Eq. (2.21) as $(w / 3 !) \int d \mathbf{r} \rho^{3}(\mathbf{r})$, allowing a decoupling of the chains by the introduction of the operator $A_{3}$ as in Eqs. (2.22) and (2.23). With this decoupling, the summations in the grand partition function (2.56) may be done explicitly, yielding

$$
\Xi_{0}=\left.\exp \left[-A_{3}(\{\delta / \delta J\})\right] \exp \left(\sum_{l} z_{0, l} Q\left(N_{l}, J\right)\right)\right|_{J=0},
$$

where the single-chain partition function Eq. (2.25) becomes

$$
\begin{aligned}
Q\left(N_{l}, J\right)= & \int \mathscr{D}[\mathbf{r}] \exp \left\{-\frac{1}{2} \int_{0}^{N_{l}} d \tau|\dot{\mathbf{r}}(\tau)|^{2}\right. \\
& \left.+\int d \mathbf{r} \int_{0}^{N_{l}} d \tau J(\mathbf{r}) \delta[\mathbf{r}-\mathbf{r}(\tau)]\right\} .
\end{aligned}
$$

Generalizing Eqs. (2.27) and (2.28) in the monodisperse case, the effect of the operator $A_{3}$ can be written as

$$
\begin{aligned}
e^{-A_{3}} & \exp \left(\sum_{l} z_{0, l} Q_{l}\right) \\
= & \sum_{n=0}^{\infty} \frac{1}{n !} \sum_{l_{1}} \cdots \sum_{l_{l}} \prod_{i=1}^{n} z_{0, l_{l}}\left\langle Q_{l_{1}} \cdots Q_{l_{n}}\right\rangle,
\end{aligned}
$$

with the abbreviated notation $Q_{l}=Q\left(N_{l}, J\right)$. To first order in the three-body excluded volume amplitude, the contribution of the term of order $Q^{n}$ in the expansion of (B1) is

$$
\begin{aligned}
\left\langle Q_{l_{1}} \cdots Q_{l_{n}}\right\rangle= & V^{n}\left\{1-w\left[\sum_{i=1}^{n} P_{1}\left(N_{l_{i}}\right)+\sum_{i \neq j}^{n} P_{2}\left(N_{l_{l}}, N_{l_{l}}\right)\right.\right. \\
& \left.\left.+\sum_{i \neq j \neq k}^{n} P_{3}\left(N_{l_{i}}, N_{l_{j}}, N_{l_{k}}\right)\right]+O\left(w^{2}\right)\right\} .
\end{aligned}
$$


The $m$ chain diagrams whose values are represented by the quantities $P_{m}$ are the same as in Fig. 2, but with distinct polymerization indices for each of the chains. By the computational rules discussed in Appendix A, and by explicit summation of the series in (B3), we find to first order in $w$

$$
\begin{aligned}
\Xi_{0} \approx & \exp \left(V \sum_{j} z_{0, j}\right)\left\{1-w V\left[p_{1} \sum_{k} z_{0, k} N_{k}^{3-d}\right.\right. \\
& +p_{2} \sum_{k, l} z_{0, k} z_{0, l} N_{l} N_{m}^{2-d / 2} \\
& \left.\left.+p_{3} \sum_{k, l, m} z_{0, k} z_{0, l} z_{0, m} N_{k} N_{l} N_{m}\right]+O\left(w^{2}\right)\right\} .
\end{aligned}
$$

Introducing the total reference fugacity $\bar{z}_{0}=\Sigma_{l} z_{0, l}$ and fugacity moments $\left\langle N^{\alpha}\right\rangle=\bar{z}_{0}^{-1} \Sigma_{l} z_{0, l} N_{l}^{\alpha}$, we obtain the final result quoted in Eq. (2.61).

'I. C. Sanchez, J. Appl. Phys. 58, 2871 (1985).

${ }^{2}$ K. Shinozaki, T. van Tan, Y. Saito, and T. Nose, Polymer 23, 728 ( 1982 ).

${ }^{3}$ T. Dobashi, M. Nakata, and M. Kaneko, J. Chem. Phys. 72, 6185, 6692 (1980); 80, 948 (1984).

${ }^{4}$ P. J. Flory, Principles of Polymer Chemistry (Cornell University, Ithaca, 1979).

${ }^{5}$ A. Vrij and G. J. Roebersen, J. Polymer Sci. Polymer Phys. Ed. 15, 109 (1977).

'T. Nose, Polymer J. 8, 96 ( 1976).

${ }^{7}$ K. Shinozaki and T. Nose, Polymer J. (Japan) 13, 1119 (1981); K. Shinozaki, T. Hamada, and T. Nose, J. Chem. Phys. 77, 4734 (1982).

${ }^{8}$ S. F. Edwards, Proc. Phys. Soc. London 88, 900 (1984).

${ }^{9}$ The derivation of an LGW Hamiltonian from a continuum model of polymer solutions in good solvents, those for which the binary excluded-volume parameter $v>0$, is discussed in T. Ohta and A. Nakanishi, J. Phys. A: Math. Gen. 16, 4155 (1983).

${ }^{10} \mathrm{~J}$. Hubbard and P. Schofield, Phys. Lett. A 40, 245 (1972).

${ }^{11}$ R. E. Goldstein and A. Parola, J. Chem. Phys. 88, 7059 (1988).

${ }^{12}$ R. Zwanzig, J. Chem. Phys. 22, 1420 (1954).

${ }^{13}$ J. D. Weeks, D. Chandler, and H. C. Andersen, J. Chem. Phys. 54, 5237 (1971).

${ }^{14}$ H. Yamakawa, Modern Theory of Polymer Solutions (Harper and Row, New York, 1971), and references therein.

${ }^{15}$ The possible role of three-body excluded-volume interactions in affecting the molecular weight dependence of the critical volume fraction has been explored in M. Muthukumar, J. Chem. Phys. 85, 4722 (1986).

${ }^{16}$ Renormalization group treatments (Refs. 22,23) of the theta point in polymer solutions show in fact that $T_{\theta}$ of the finite chain system actually occurs below the temperature at which the two-body amplitude $v(T)$ vanishes, so that the ideal chain behavior arises from a competition of attractive two-body and repulsive three-body interactions.

${ }^{17}$ R. E. Goldstein, A. Parola, N. W. Ashcroft, M. W. Pestak, M. H. W. Chan, J. R. de Bruyn, and D. A. Balzarini, Phys. Rev. Lett. 58, 41 (1987); M. W. Pestak, R. E. Goldstein, M. H. W. Chan, J. R. de Bruyn, D. A. Balzarini, and N. W. Ashcroft, Phys. Rev. B 36, 599 (1987).

${ }^{18}$ R. Kubo, J. Phys. Soc. Jpn. 17, 1100 (1972).

${ }^{19} \mathrm{G}$. Stell, in Phase Transitions and Critical Phenomena, edited by C. Domb and M. S. Green (Academic, New York, 1976), Vol. 5b, p. 205.

${ }^{20}$ P. J. Flory and H. Daoust, J. Polymer Sci. 25, 429 (1957).

${ }^{21}$ P. G. de Gennes, Scaling Concepts in Polymer Physics (Cornell University, Ithaca, 1979).

${ }^{22}$ B. J. Cherayil, J. F. Douglas, and K. F. Freed, J. Chem. Phys. 83, 5293 (1985).

${ }^{23}$ B. J. Cherayil, A. L. Kholodenko, and K. F. Freed, J. Chem. Phys. 86, 7204 (1987).

${ }^{24} \mathrm{D}$. Amit, Field Theory, The Renormalization Group and Critical Phenomena (McGraw-Hill, London, 1978).

${ }^{25}$ See, for instance, K. F. Freed, Renormalization Group Theory of Macromolecules (Wiley, New York, 1987).

${ }^{26} \mathrm{We}$ are grateful to A. Parola for important insights into the cumulant structure of fluids.

${ }^{27}$ I. C. Sanchez, Macromolecules 17, 967 (1984).

${ }^{28}$ W. H. Stockmayer, J. Chem. Phys. 17, 588 (1949).

${ }^{29}$ B. Widom, J. Stat. Phys. 52, 1343 (1988); 53, 523 (1988).

${ }^{30}$ S. Stepanow, J. Physique 48, 2037 (1987).

${ }^{31}$ J. S. Rowlinson and B. Widom, Molecular Theory of Capillarity (Oxford, New York, 1982).

${ }^{32}$ Reference 21, p. 213; P. G. de Gennes, Phys. Lett. A 26, 313 (1968).

${ }^{33}$ A. I. Pesci, R. E. Goldstein, and K. F. Freed (unpublished).

${ }^{34}$ K. F. Freed, J. Phys. A 18, 871 (1985).

${ }^{35}$ M. G. Bawendi, K. F. Freed, and U. Mohanty, J. Chem. Phys. 87, 5534 (1987).

${ }^{36}$ P. G. de Gennes, Phys. Lett. A 38, 339 (1972).

${ }^{37}$ R. E. Goldstein and B. J. Cherayil (unpublished).

${ }^{38}$ For a recent application of Gaussian fluctuation theory to polymer solutions, see M. O. de la Cruz, S. F. Edwards, and I. C. Sanchez, J. Chem. Phys. 89, 1704 (1988).

${ }^{39}$ E. Brézin and S. Feng, Phys. Rev. B 29, 472 (1984).

${ }^{40}$ Some aspects of the statistical mechanics of nonsymmetric LGW Hamiltonians have been explored in J. F. Nicoll, Phys. Rev. A 24, 2203 (1981). See also Ref. 11.

${ }^{41}$ See, e.g., P. Ramond, Field Theory, A Modern Primer (Benjamin/Cummings, Reading, Massachusetts, 1981), p. 157. 